\title{
Diacronie
}

Studi di Storia Contemporanea

$N^{\circ} 27,3$ | 2016

Stato, costituzione e democrazia

\section{La rete nel Baltico. Percorsi di ricerca in uno spazio sospeso fra Est e Ovest}

\section{Deborah Paci}

\section{(C) OpenEdition}

\section{Journals}

\section{Edizione digitale}

URL: http://journals.openedition.org/diacronie/4315

DOI: $10.4000 /$ diacronie.4315

ISSN: 2038-0925

\section{Editore}

Association culturelle Diacronie

\section{Notizia bibliografica digitale}

Deborah Paci, «La rete nel Baltico. Percorsi di ricerca in uno spazio sospeso fra Est e Ovest », Diacronie [Online], № 27, 3 | 2016, documento 13, Messo online il 29 septembre 2016, consultato il 30 avril 2019. URL : http://journals.openedition.org/diacronie/4315; DOI : 10.4000/diacronie.4315 


\title{
Diacronie
}

\section{La rete nel Baltico. Percorsi di ricerca in uno spazio sospeso fra Est e Ovest}

\author{
Deborah PACI *
}

Questa rassegna dei siti web relativi ai centri di ricerca specializzati sulla regione del Mar Baltico intende offrire un quadro di insieme delle attività animate da esperti $e$ studiosi che condividono un interesse nell'evoluzione dei rapporti tra l'Unione Europea e i paesi che si affacciano sul Mar Baltico. Un'attenzione particolare è rivolta alle iniziative congiunte, incluse la presentazione di conferenze accademiche, all'organizzazione di workshop e programmi di studio post-laurea.

\section{Introduzione}

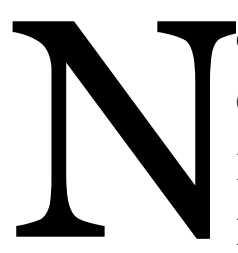

ove Paesi dai differenti retaggi storici, tradizioni culturali e linguistiche Germania, Danimarca, Svezia, Finlandia, Russia, Estonia, Lettonia, Lituania e Polonia - si affacciano sul grande mare interno del Nord Europa, il Baltico. Insieme costituiscono una peculiare entità spaziale che è la cosiddetta regione del mar Baltico․ A lungo questa regione è stato un luogo di confronto - spesso conflittuale - per il dominio dell'area, fin dai secoli XVI e XVII quando Svezia e Danimarca vennero allo scontro per il controllo di quel mare. Nel contesto del dominium maris Baltici la Svezia divenne una grande potenza europea e il Baltico il mare interno svedese. Nel XVIII secolo l'Impero russo fu il principale contendente della Svezia a cui fu tolta la primazia sul Baltico nel XIX secolo. Nel

\footnotetext{
${ }^{1}$ Sulla storia della regione baltica in prospettiva diacronica si veda la recente raccolta di saggi: MOTTA, Giovanna (a cura di), Il Baltico: Un mare interno nella storia di lungo periodo, Roma, Edizioni Nuova Cultura, 2013. Sull'immaginario identitario nella regione del Mar Baltico si veda GÖTZ, Norbert (edited by), The Sea of Identities. A Century of Baltic and East European Experiences with Nationality, Class and Gender, Huddinge, Södertörn University, 2014.
} 
Novecento la cortina di ferro, quella linea divisoria che correva lungo il Baltico, avrebbe separato l'Est dall'Ovest. Il crollo di quel muro e la fine della Guerra Fredda rappresentò un momento di cesura nella storia della regione baltica. Risorse quell'idea di unità nello spirito di cooperazione che risaliva ai tempi della Lega Anseatica, nel XIII secolo, e che era divenuta tangibile sul piano politico nell'Unione di Kalmar che, nei secoli XV e XVI, unificò i tre regni di Danimarca, Svezia e Norvegia sotto un'unica entità sovrana. I centri di ricerca che sono sorti negli ultimi dieci anni riflettono la tendenza a voler rappresentare la regione baltica come una grande comunità unitaria e cooperativa, contraddistinta da uno spiccato dinamismo culturale ${ }^{2}$.

\section{La cooperazione nella regione del Mar Baltico: BalticStudyNet, Centre for Baltic and East European Studies, «Baltic Worlds»}

BalticStudyNet - Promoting Baltic Sea Region Higher Education worldwide è un programma di ricerca e di promozione culturale dell'area baltica nel quadro dell'Erasmus Mundus della Commissione europea: è stato condotto, dal 2006 al 2009, da un consorzio di otto università che si affacciano sulle rive del mar Baltico (Germania, Danimarca, Svezia, Finlandia, Estonia, Lettonia, Lituania, Polonia). Tale programma è stato coordinato dal Professor Bernd Henningsen del dipartimento di studi nordeuropei dell'Università Humboldt di Berlino. Sono state avviate diverse iniziative a partire dall'organizzazioni di conferenze 3 e tour promozionali in Russia, Ucraina, Stati Uniti, Cina, India, Armenia e Australia4. Risulta di particolare interesse la sezione del sito dedicata alle pubblicazioni consultabili liberamente e gratuitamente riguardanti la regione baltica in un contesto globales.

Il Centre for Baltic and East European Studies (CBEES) afferente alla Södertörns högskola di Stoccolma è sorto nel 2005 allo scopo di promuovere e sviluppare le ricerche sulla regione del mar Baltico e sull'Europa Orientale, a partire da quelle condotte dai dottoranti affiliati alla Baltic and East European Graduate School

\footnotetext{
2 Sul panorama degli studi e dei centri di ricerca relativi alla regione baltica si rimanda a SCHYMIK, Carsten, HENZE, Valeska, HILLE, Jochen (edited by), Go North! Baltic Sea Region Studies: Past-Present-Future, Berlin, BWV Verlag, 2011.

3 URL: < http://www2.hu-berlin.de/balticstudynet/events.html > [consultato il 15 settembre 2016].

4 URL: < http://www2.hu-berlin.de/balticstudynet/worldtour.htm > [consultato il 15 settembre 2016].

5 URL: < http://www2.hu-berlin.de/balticstudynet/publications.html > [consultato il 15 settembre 2016].
} 
(BEEGS) ${ }^{6}$. Annualmente il CBEES accoglie ricercatori invitati ${ }^{7}$ e periodicamente anima seminari e conferenze internazionali a cui sono chiamati a partecipare non solo studiosi accademici, ma anche quanti intendano portare queste tematiche all'attenzione di un pubblico più vasto dei soli addetti ai lavori. La cooperazione con altri centri di ricerca è un tratto distintivo del CBEES: lo dimostra la fitta rete di rapporti di collaborazione con studiosi esterni al centro ${ }^{8}$. La newsletter dà conto di tutte le attività animate dal CBEES9. I temi che sono attualmente al centro dell'interesse del CBEES sono: le politiche spaziali ${ }^{10}$, identità e cittadinanza ${ }^{11}$, memoria e retaggio ${ }^{12}$. Il fiore all'occhiello delle attività del CBEES è la conferenza annuale, che quest'anno si terrà ai primi di dicembre e affronterà il tema delle trasformazioni spaziali e culturali nell'area baltica secondo l'approccio proprio degli area studies. "Transforming Spaces - Mastering Uncertainty: A second Take on Area Studies in the Baltic Sea region and Eastern Europe”, questo è il titolo della conferenza organizzata dal CBEES che vedrà la partecipazione in qualità di keynote di Teresa Kulawik (Södertörns högskola), di Andrea Petö (Central European University - CEU di Budapest) e di James Wesley Scott (University of Eastern Finland di Joensuu) ${ }^{13}$. Di particolare interesse è la sezione del

\footnotetext{
6 URL:

< http://www.sh.se/p3/ext/content.nsf/aget?openagent\&key=about_us_1301902860317 > [consultato il 15 settembre 2016].

7 URL:

<http://www.sh.se/p3/ext/content.nsf/aget?openagent\&key=about_us_1301902860317\#!/p3/ ext/content.nsf/aget?openagent\&key=visiting_researchers_1304062546566 > [consultato il 15 settembre 2016].

8 URL:

<http://www.sh.se/p3/ext/content.nsf/aget?openagent\&key=about_us_1301902860317\#!/p3/ ext/content.nsf/aget?openagent\&key=external_relations_1301925754712> [consultato il 15 settembre 2016].

9URL:

<http://www.sh.se/p3/ext/content.nsf/aget?openagent\&key=about_us_1301902860317\#!/p3/ ext/content.nsf/aget?openagent\&key=newsletters_and_information_1304069654284 > [consultato il 15 settembre 2016].

10 URL:

<http://www.sh.se/p3/ext/content.nsf/aget?openagent\&key=about_us_1301902860317\#!/p3/ ext/content.nsf/aget?openagent\&key=politics_of_space_1458142241151 > [consultato il 15 settembre 2016].

${ }^{11}$ URL:

<http://www.sh.se/p3/ext/content.nsf/aget?openagent\&key=about_us_1301902860317\#!/p3/ ext/content.nsf/aget?openagent\&key=identity_and_citizenship_1458142406890 > [consultato il 15 settembre 2016].

12 URL:

<http://www.sh.se/p3/ext/content.nsf/aget?openagent\&key=about_us_1301902860317\#!/p3/ ext/content.nsf/aget?openagent\&key=memory_and_heritage_1458142839988 > [consultato il 15 settembre 2016].

13URL:

<http://www.sh.se/p3/ext/content.nsf/aget?openagent\&key=sh_konferens_o_en_1461052557 778\#!/p3/ext/content.nsf/aget?openagent\&key=sh_konferens_o_en_1461052557778 > [consultato il 15 settembre 2016].
} 
sito dove è possibile consultare le pubblicazioni a firma degli associati al centro nonché le tesi di dottorato degli studenti afferenti al BEEGS ${ }^{14}$.

Le ricerche condotte dagli affiliati al CBEES trovano spazio sulla rivista trimestrale open access «Baltic Worlds». Sorta nel 2008 potendo contare sul finanziamento della Östersjöstiftelsen ${ }^{15}$ «Baltic Worlds» ospita $\operatorname{articoli~}^{16}$ e recensioni ${ }^{17}$ sottoposti ad un processo di referaggio da parte di un comitato scientifico che si compone di studiosi provenienti da differenti aree disciplinari. L'obiettivo della rivista è quello di far dialogare studiosi specialisti in scienze economiche, politiche e storiche della regione baltica, dell'Europa orientale, dell'area caucasica e balcanica allo scopo di rappresentare un punto di riferimento delle ricerche incentrate su quelle aree. Ogni numero della rivista in versione integrale è consultabile e scaricabile in formato $\mathrm{pdf}^{18}$. Il portale della rivista ospita una selezione di saggi precedentemente pubblicati sulla rivista. In aggiunta alla versione elettronica è prevista la pubblicazione di una versione cartacea, che viene inviata a coloro i quali sottoscrivono un abbonamento annuale (gratuito o a pagamento) ${ }^{19}$.

\section{Il Baltico, le relazioni tra l'Unione Europea e la Federazione Russa: il CEURUS}

Le complesse relazioni tra l'Unione Europea e la Federazione Russa sono al centro dell'agenda politica dell'Unione Europea da più di un decennio, in particolare dal 1999 quando, nell'ambito della Northern Dimension, le istituzioni europee hanno perseguito l'obiettivo di promuovere il dialogo politico ed energetico, la cooperazione economica,

\footnotetext{
14 URL:

<http://www.sh.se/p3/ext/content.nsf/aget?openagent\&key=about_us_1301902860317\#!/p3/ ext/content.nsf/aget?openagent\&key=sh_publikationer_inst_en_3200o $>$ [consultato il 15 settembre 2016].

${ }^{15}$ La Östersjöstiftelsen (Fondazione per gli studi baltici ed est-europei) è stata fondata nel 1994 per iniziativa del governo svedese allo scopo di sostenere l'attività di ricerca in scienze naturali, umane e sociali, a partire dalle borse di studio rilasciate ai dottorandi afferenti alla Södertörns högskola di Stoccolma. Da allora la Östersjöstiftelsen ha elargito circa 250 milioni di euro a supporto di approssimativamente 160 progetti di ricerca e 115 dottorandi. Tra le ricerche finanziate dalla Östersjöstiftelsen vi è quella di cui è capofila un affiliato al CBEES, il professor Norbert Götz, che coordina in stretta collaborazione con l'Università Ca' Foscari di Venezia - di cui il referente è il professor Rolf Petri - il progetto "Spaces of Expectation: Mental Mapping and Historical Imagination in the Baltic Sea and Mediterranean Region". Tale progetto si propone di indagare in chiave comparata l'immaginario storico regionale nell'area baltica e mediterranea seguendo l'approccio metodologico proprio degli area studies, mental maps e conceptual history.

${ }^{16}$ URL: < http://balticworlds.com/category/essays/ > [consultato il 15 settembre 2016].

17 URL: < http://balticworlds.com/category/reviews/ > [consultato il 15 settembre 2016].

${ }_{18}$ URL: < http://balticworlds.com/back-issues/ > [consultato il 15 settembre 2016].

19 URL: < http://balticworlds.com/subscribe/ > [consultato il 15 settembre 2016].
} 
in campo culturale, tecnologico e in materia di giustizia cercando di privilegiare gli aspetti legati alla politica internazionale e al tema dell'ambiente ${ }^{20}$.

Il Centre for EU Russia Studies (CEURUS) è un centro di ricerca e di insegnamento afferente all'Università di Tartu, in Estonia, che privilegia temi a carattere multidisciplinare e transdisciplinare inerenti alle relazioni UE/Federazione Russa ${ }^{21}$. Si contraddistingue per la sua propensione a rappresentare un luogo ideale di incontro e di discussione rivolto a studenti e studiosi che condividono un interesse precipuo per queste tematiche. Nato come evoluzione dei programmi di cerca e di studio legati principalmente all'Institute of Government and Politics (IGP) e all'EuroCollege (EC), Il CEURUS è stato costituito nel 2011 nell'ambito del programma di ricerca e sviluppo finanziato dall'Università di Tartu. Le finalità del CEURUS possono essere riassunti nei seguenti punti: lo sviluppo di ricerche di alta qualità sotto il profilo scientifico che si combinino con una conoscenza accurata degli studi inerenti all'area interessata; un investimento significativo sui programmi di studio a tutti i livelli della formazione universitaria; la conversione della ricerca pura alla ricerca applicata che consente di fornire indicazioni utili riguardanti gli aspetti politici delle relazioni tra l'Unione Europea e la Federazione Russa; la valorizzazione dei risultati della ricerca al fine di incrementare e stimolare il dibattito pubblico ${ }^{22}$. Al CEURUS afferiscono sei gruppi di ricerca di eccellenza che possono godere, nella maggior parte dei casi, del finanziamento rilasciato dalla Fondazione della scienza estone: "Governance \& Democrazia" coordinato dal Professor Viatcheslav Morozov ambisce a indagare lo sviluppo dei regimi politici, i modelli di rappresentanza e di legittimazione politica nonché le strutture di governance sia nella UE sia nella Federazione Russa, prendendo in esame le trasformazioni ideologiche intervenute nelle due entità politica in chiave comparativa; "Confini, regioni \& quartieri” sotto la responsabilità del Professor Andrey Makarychev pone l'attenzione sulla questione dei confini nel dibattito pubblico e politico; "Energia e Ambiente" animato dal Professor Andrei Belyi si propone di esaminare la politica energetica nella UE e nella Federazione Russa analizzando le sfide

${ }^{20}$ Sulle relazioni tra Unione Europea e Russia si vedano, tra gli altri: JOHNSON, Debra, ROBINSON, Paul (edited by), Perspectives on Eu-Russia relations, London, Routledge, 2005; GOMART, Thomas, Eu-Russia relations : toward a way out of depression, Washington DC, Center for strategic \& international studies, 2008; BELYAEVA, Nina (edited by), Russia and the EU: uneasy relations : a look from Belgium : academic papers of the 9th International Session of the HSE Russian - European Centre for multidisciplinary research, Brugge, 2-9 August, 2009, Kaunas, Vytautas Magnus University press, 2010; PROZOROV, Sergei, Understanding conflict between Russia and the EU : the limits of integration, Houndmills-New York, Palgrave, 2006.

${ }^{21}$ URL: < http://ceurus.ut.ee/_> [consultato il 15 settembre 2016].

22 URL: < http://ceurus.ut.ee/home/about/mission-2/ > [consultato il 15 settembre 2016]. 
nel mercato internazionale del gas, le conseguenze dello sviluppo del gas da argille e l'impatto della liberalizzazione del gas in Europa e in Russia; "Storia e Memoria" sotto la direzione di Maria Mälksoo ambisce ad analizzare il rapporto tra storia e memoria con un riferimento particolare rivolto a ciò che rimane nell'immaginario collettivo europeo del ricordo della Seconda Guerra Mondiale e del comunismo sovietico, attraverso un'indagine volta a comprendere i processi di politicizzazione della storia in Russia e in alcuni paesi della UE; "Sovranità, sicurezza e ordine" coordinato da Eiki Berg pone al centro dell'analisi le questioni legate alle norme sulla sovranità e all'integrità territoriale alla luce dei risvolti sul piano politico e giuridico verificatisi a seguito della guerra russo-georgiana e della dichiarazione unilaterale di indipendenza del Kosovo. "Diritto internazionale \& diritti umani” diretto da Lauri Mälksoo esamina le modalità con cui la Federazione Russa applica il diritto pubblico internazionale e si pone di fronte alla legislazione europea sui diritti umani, investigando i diversi fattori (un lungo trascorso imperiale e la mancanza di una tradizione liberale) che rendono difficoltosa la comprensione del diritto internazionale ${ }^{23}$.

Il CEURUS promuove forme di collaborazione nel quadro di visiting fellowships tra studiosi provenienti da realtà accademiche europee e russe e favorisce la mobilità di studenti internazionali all'interno di programmi di studio specifici. Gli studiosi affiliati al CEURUS dirigono le ricerche dei dottorandi ${ }^{24}$ che al termine del dottorato possono profittare di fellowships ${ }^{25}$ e partecipano all'attività didattica nei corsi di Master dell'Università di Tartu e in particolare nel programma di Master in EU-Russia Studies coordinato dall'EuroCollege ${ }^{26}$.

Il CEURUS fa parte dell'UPTAKE consortium, insieme all'Uppsala Centre for Russian and Eurasian Studies dell'Università di Uppsala e al Global Europe Centre dell'University of Kent, il consorzio finanziato dal programma europeo Horizon 2020 sotto la denominazione "Building Research Excellence in Russian and East European Studies at the Universities of Tartu, Uppsala and Kent" che promuove progetti di eccellenza nel campo degli studi sulla Russia e sui paesi dell'Europa Orientale attraverso l'organizzazione di conferenze e di corsi di insegnamento rivolti a neolaureati nonché la supervisione di tesi di dottorato e di ricerche post-dottorali ${ }^{27}$. Il

23 URL: < http://ceurus.ut.ee/home/home/research/research-cluster/ > [consultato il 15 settembre 2016].

24 URL: < http://ceurus.ut.ee/home/study/phd-with-ceurus/ > [consultato il 15 settembre 2016].

25 URL: < http://ceurus.ut.ee/home/fellowships/ > [consultato il 15 settembre 2016].

26 URL: < http://ceurus.ut.ee/home/study/ma-programs/\#Baltic\%20Sea > [consultato il 15 settembre 2016].

27 URL: < http://www.uptake.ut.ee/ > [consultato il 15 settembre 2016]. 
CEURUS pubblica il "EU-Russia Watch", un rapporto annuale relativo alle relazioni tra i membri dell'Unione Europea e la Federazione Russa. Sul sito è possibile consultare il rapporto del 2012 dove l'attenzione è concentrata sull'evoluzione delle relazioni bilaterali tra UE e Federazione Russa che sono intervenute nel corso del 201128. Anche l'EU-Russia Paper Series fa capo al CEURUS: fondato nel settembre 2011 ospita i contributi degli studiosi che afferiscono al centro liberamente consultabili sul sito ${ }^{29}$.

\section{Il Baltico, area di pace: SIPRI e Åland fredsinstitut}

La regione del Mar Baltico - per eccellenza area di pace e di risoluzione dei conflitti - è una determinazione identitaria che è stata sostenuta con forza non soltanto dai policymakers dell'area baltica ma anche da larga parte dei politologi e dai media. Non è un caso che proprio in Svezia, a Stoccolma, operi dal 1966 lo Stockholm International Peace Research Institute (SIPRI) un istituto internazionale indipendente, supportato sotto il profilo economico dal governo svedese, la cui attività principale consiste nella conduzione di ricerche relative ai conflitti, agli armamenti e al controllo delle armi in un contesto globale. Il SIPRI ha una sua rappresentanza a Pechino e coopera in maniera assidua con organizzazioni intergovernative: le Nazioni Unite, l'Unione Europea; riceve inoltre, periodicamente, ricercatori invitati così come delegazioni accademiche e governative.

Un altro centro di studi che si vuole proporre come laboratorio privilegiato dove poter condurre ricerche su temi connessi con la sicurezza, la pace, i conflitti e i diritti delle minoranze è l'ي̊lands fredsinstitut. Sorto nel 1992 a Mariehamn, nelle isole Åland, l'Åland fredsinstitut è una fondazione caritatevole indipendente che svolge numerose attività di ricerca in cooperazione con altre organizzazioni nazionali e internazionali partendo dalla considerazione che lo statuto dell'arcipelago alandese - regione demilitarizzata svedesofona all'interno dello stato finlandese, dotata di un diritto all'autogoverno assicurato da specifiche garanzie internazionali che risalgono al $1922^{30}$

\footnotetext{
${ }^{28}$ URL: < http://ceurus.ut.ee/home/eu-russia-watch/ > [consultato il 15 settembre 2016].

29 URL: < http://ceurus.ut.ee/home/eu-russia-papers/ > [consultato il 15 settembre 2016].

${ }^{30}$ Sulle isole Åland si rimanda, tra gli altri, a BARROS, James, The Alland Islands Question: Its Settlement by the League of Nations, New Haven-London, Yale University Press, 1968; ERIKSSON, Susanne, JOHANSSON, Lars Ingmar, SUNDBACK, Barbro, The Åland Islands demilitarized region, Mariehamn, Ålands fredsförening, 1995; ERIKSSON, Susanne, JOHANSSON, Lars Ingmar, SUNDBACK, Barbro, Islands of Peace. Aland's autonomy, demilitarisation and neutralisation, Mariehamn, The Åland Islands Peace Institute, 2006; CHILlAUD, Matthieu (sous la dir. de), Les Îles Åland en mer Baltique. Héritage et actualité d'un régime original, Paris, L'Harmattan, 2009; EDQUIST, Samuel, HOLMÉN, Janne, Islands of Identity. History-writing and identity formation in five island regions in the Baltic Sea,
} 
- rappresenti un modello utile ad altre realtà con caratteristiche simili31. La dimensione e la rilevanza sul piano internazionale di cui gode l'Åland fredsinstitut è comprovata dai rapporti assidui che l'istituto intrattiene con studiosi, giornalisti, politici provenienti dal Caucaso meridionale, Giappone, Cina, Medioriente, Balcani, Iran, Centro America. Le sezioni più interessanti e utili del sito sono quelle relative alle risorse liberamente consultabili relative alla storia e all'attualità del modello alandese e più in generale ai temi legati ai diritti delle minoranze etniche e linguistiche ${ }^{2}$.

Huddinge, Södertörns University, 2015; PACI, Deborah, L'arcipelago della pace. Le isole Åland e il Baltico (XIX-XXI sec.), Milano, Unicopli, 2016.

${ }^{31}$ URL: < http://www.peace.ax/en/the-aland-example > [consultato il 15 settembre 2016].

32 URL: < http://www.peace.ax/en/the-aland-example/resources > [consultato il 15 settembre 2016]; URL: < http://www.peace.ax/en/publications > [consultato il 15 settembre 2016]. 


\section{${ }^{*}$ L'autore}

Deborah Paci è assegnista di ricerca presso il Dipartimento di Studi linguistici e culturali comparati dell'Università Ca' Foscari di Venezia e ricercatrice associata al Centre de la Méditerranée Moderne et Contemporaine dell'Université de Nice Sophia Antipolis. È autrice delle monografie: L'arcipelago della pace. Le isole Åland e il Baltico (XIX-XXI sec.), Milano, Unicopli, 2016; Corsica fatal, Malta baluardo di romanità. L'irredentismo fascista nel Mare nostrum (1922-1942), Firenze, Le Monnier-Mondadori Education, 2015.

URL: < http://www.studistorici.com/2009/o2/24/deborah-paci/ >

\section{Per citare questo articolo:}

PACl, Deborah, «La rete nel Baltico. Percorsi di ricerca in uno spazio sospeso fra Est e Ovest», Diacronie. Studi di Storia Contemporanea : Stato, costituzione e democrazia, 29/09/2016,

URL:< http://www.studistorici.com/2016/09/29/paci_numero_27/ >

Diacronie Studi di Storia Contemporanea $\vartheta$ www.diacronie.it

Risorsa digitale indipendente a carattere storiografico. Uscita trimestrale. redazione.diacronie@hotmail.it

Comitato di redazione: Jacopo Bassi - Luca Bufarale - Elisa Grandi - Antonio César Moreno Cantano - Deborah Paci - Fausto Pietrancosta - Alessandro Salvador - Matteo Tomasoni - Luca Zuccolo

Diritti: gli articoli di Diacronie. Studi di Storia Contemporanea sono pubblicati sotto licenza Creative Commons 3.0. Possono essere riprodotti e modificati a patto di indicare eventuali modifiche dei contenuti, di riconoscere la paternità dell'opera e di condividerla allo stesso modo. La citazione di estratti è comunque sempre autorizzata, nei limiti previsti dalla legge. 